\title{
The Comparison of Reliability of the Hypercube and Crossed Cube Wang Dong-xia ${ }^{1, a}$, Qin Qiong ${ }^{2, b}$, Lu Hui-min ${ }^{3, c}$ \\ ${ }^{1}$ Dept of Basic Science, Dalian Naval Academy, Dalian 116018, China \\ 2 Dept of Basic Science, Dalian Naval Academy, Dalian 116018, China \\ ${ }^{3}$ Dept of Basic Science, Dalian Naval Academy, Dalian 116018, China
}

Keywords: hypercube; crossed cube; reliability

\begin{abstract}
The crossed cube is a variation of the hypercube. In this paper, we supply the topological property of the two network structures, and compare the all-terminal reliability and node-connected reliability of them. It is obtained that the crossed cube is superior to the hypercube in the lower bound of two kinds of reliability.
\end{abstract}

\section{Introduction}

The hypercube network $\boldsymbol{Q}_{n}$ has become one of the most popular interconnection networks for its simple structure and nice properties. The crossed cube $\boldsymbol{C Q}_{\boldsymbol{n}}$ is an attractive alternative for the ordinary hypercube. It is a more generalized version of the twisted hypercube which is obtained by interchanging a pair of edges of the ordinary hypercube. Both the crossed cube and the ordinary hypercube have the same number of vertices and the same node degree. Recent research studies have revealed that the crossed cube, as an interconnection network, possesses many desirable properties which are not available in ordinary hypercube [1]. The crossed cube has a half diameter of the hypercube approximately [2]. Also it contains the optimal complete binary tree as subgraph whereas the ordinary hypercube does not[3].

In this paper, we supply the topological property of the two network structures by proving lower bounds of all-terminal reliability and node-connected reliability, and draw a conclusion that the crossed cube is superior to the hypercube in the lower bound of two kinds of reliability.

\section{Basic definitions}

The n-dimensional hypercube $\boldsymbol{Q}_{n}$ and the n-dimensional crossed cube $\boldsymbol{C}_{\boldsymbol{n}}$ are n-regular graphs with $\mathbf{2}^{\boldsymbol{n}}$ vertices and $\mathbf{2}^{\boldsymbol{n}-\mathbf{n}} \boldsymbol{n}$ edges. A vertex in $\boldsymbol{C} \boldsymbol{Q}_{n}$ or $\boldsymbol{Q}_{\boldsymbol{n}}$ will be represented by a binary string $v_{n-1} v_{n-2} \cdots v_{1} v_{0}$ of length $n$ in $Q_{n}$, the string is defined to be the address of the vertex, two vertices are adjacent if and only if their binary addresses differ only in one bit position .

Definition 1.The n-dimensional crossed cube $\boldsymbol{C Q}_{\boldsymbol{n}}$ is the labeled graph defined recursively as follows.

$\boldsymbol{C Q}_{1}$ is the complete graph of two vertices labeled by 0 and 1 .

For $\boldsymbol{n} \geq 2, \boldsymbol{C} \boldsymbol{Q}_{n}$ is obtained by taking two copies of $\boldsymbol{C} \boldsymbol{Q}_{n-1}$, denoted by $\boldsymbol{C Q}_{n-1}^{0}$ with vertex-set

$V\left(C Q_{n-1}^{0}\right)=\left\{0 x_{n-2} x_{n-3} \cdots x_{0}: x_{i}=0\right.$ or 1$\}$

and $C Q_{n-1}^{1}$ with vertex-set

$V\left(C Q_{n-1}^{1}\right)=\left\{0 x_{n-2} x_{n-3} \cdots x_{0}: x_{i}=0\right.$ or 1$\}$

respectively, and adding an edge joining

$0 x_{n-2} x_{n-3} \cdots x_{0} \in V\left(C Q_{n-1}^{0}\right)$

and $1 y_{n-2} y_{n-3} \cdots y_{0} \in V\left(C Q_{n-1}^{1}\right)$

if and only if 
(1) $x_{n-2}=y_{n-2}$ if $n$ is even, and

(2) $\left(x_{2 i+1} x_{2 i} y_{2 i+1} y_{2 i}\right) \in\{(00,00),(10,10),(01,11),(11,01)\}$ for $0 \leq i \leq\left\lfloor\frac{n-1}{2}\right\rfloor$.

\section{Definition 2}

Suppose the vertices of graph $G$ never fail and the edges fail independently with probability $\boldsymbol{p}$, then the all-terminal reliability of graph $G$ is $R(G, p)=\sum_{i=1}^{m} N_{i}(G) P^{i} q^{m-i}$, where $N_{i}(G)$ is the number of connected induced subgraphs of $G$ that contain exactly r edges.

\section{Definition 3}

Suppose the edges of graph $G$ never fail and the vertices fail independently with probability $\mathbf{1}-\boldsymbol{p}$, then the node-reliability of graph $G$ is $R(G, p)=\mathbf{1}-\sum_{i=1}^{m} \boldsymbol{B}_{i}(G) P^{i} q^{m-i}$, where $B_{i}(G)$ is the number of disconnected subgraphs of $G$ that contain exactly vertices.

The graphs shown in Fig1.(a)and (b) are $\boldsymbol{Q}_{3}$ and $\boldsymbol{C Q}_{3}$ respectively.

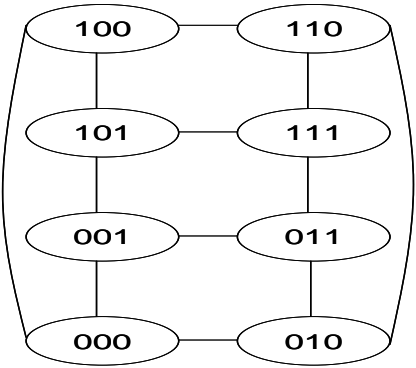

(a) $\mathbf{Q}_{3}$

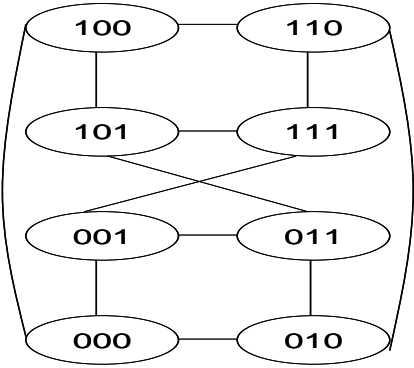

(b)CQ $\boldsymbol{Q}_{3}$

Fig.1 $(a) Q_{3}$

(b)C $\boldsymbol{Q}_{3}$

The graphs shown in Fig2.(a)and (b) are $\boldsymbol{Q}_{\mathbf{4}}$ and $\boldsymbol{C Q}_{\mathbf{4}}$,respectively. (Take graphs in Fig.1 and Fig.2 as an example. (a) is $\boldsymbol{Q}_{3}$ and (b) is $\boldsymbol{C Q}_{3}$ correspondingly. It is analogous in Fig.2)

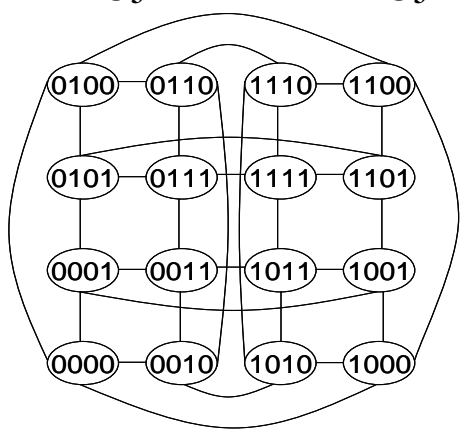

(a) $Q_{4}$

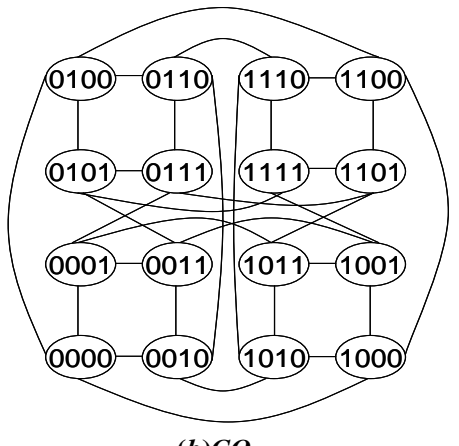

(b)CQ $Q_{4}$

Fig.2 $\quad(a) Q_{4} \quad(b) C Q_{4}$

The compare for the all terminal reliability of $Q_{n}$ and $C Q_{n}$

If $n<3, R\left(Q_{n}\right)=R\left(C Q_{n}\right)$.

If $\boldsymbol{n}=\mathbf{3}$, using factoring theorem[4], the all terminal reliability of graph $\boldsymbol{Q}_{3}$ and $\boldsymbol{C Q}_{3}$ can be obtained as follows

$$
\begin{aligned}
& R\left(Q_{3}\right)=p^{12}+12 p^{11} q+66 p^{10} q^{2}+212 p^{9} q^{3}+408 p^{8} q^{4}+384 p^{7} q^{5} \\
& R\left(C Q_{3}\right)=p^{12}+12 p^{11} q+66 p^{10} q^{2}+212 p^{9} q^{3}+409 p^{8} q^{4}+392 p^{7} q^{5}
\end{aligned}
$$

Therefore $\boldsymbol{R}\left(\boldsymbol{Q}_{3}\right) \leq \boldsymbol{R}\left(\boldsymbol{C} \boldsymbol{Q}_{3}\right)$.

If $\boldsymbol{n} \geq \mathbf{4}$, it is hard to calculate the all terminal reliability of graph $\boldsymbol{Q}_{\boldsymbol{n}}$ and $\boldsymbol{C Q}_{\boldsymbol{n}}$, but we can obtain the lower bound of all-terminal reliability because they are recursive networks from 
lower dimension to higher dimension . $\boldsymbol{Q}_{4}$ is obtained by taking two copies of $\boldsymbol{Q}_{3}$ and adding eight edges between the two copies, so we obtain the inequality of all terminal reliability of $\boldsymbol{Q}_{4}$ as follows

$$
R\left(Q_{4}\right) \geq 8 p q^{7}\left(p^{12}+12 p^{11} q+66 p^{10} q^{2}+212 p^{9} q^{3}+409 p^{8} q^{4}+392 p^{7} q^{5}\right)
$$

$\boldsymbol{C Q}_{4}$ is also obtained by taking two copies of $\boldsymbol{C Q}_{3}$ and adding eight edges in the way of definition 1 , so the inequality of all terminal reliability of $\boldsymbol{Q}_{\mathbf{4}}$ can also be obtained in the same way.

$$
R\left(C Q_{4}\right) \geq 8 p q^{7}\left(p^{12}+12 p^{11} q+66 p^{10} q^{2}+212 p^{9} q^{3}+408 p^{8} q^{4}+384 p^{7} q^{5}\right)
$$

If $n \geq 4$, we can obtain the lower bound of $R\left(Q_{n}\right)$ and the lower bound of $R\left(Q_{n}\right)$ as follows

$$
\begin{aligned}
& \left(\prod_{i=4}^{n-1} 2^{i-1} p q^{2^{i-1}-1}\right)\left(p^{12}+12 p^{11} q+66 p^{10} q^{2}+212 p^{9} q^{3}+409 p^{8} q^{4}+392 p^{7} q^{5}\right) \\
& \left(\prod_{i=4}^{n-1} 2^{i-1} p q^{2^{i-1}-1}\right)\left(p^{12}+12 p^{11} q+66 p^{10} q^{2}+212 p^{9} q^{3}+409 p^{8} q^{4}+384 p^{7} q^{5}\right)
\end{aligned}
$$

It is clear that the lower bound of $\boldsymbol{R}\left(Q_{n}\right)$ is greater than he lower bound of $R\left(C Q_{4}\right)$.

\section{The compare for the node-connected reliability of $Q_{n}$ and $C Q_{n}$}

If $n<3, R\left(Q_{n}\right)=R\left(C Q_{n}\right)$.

If $\boldsymbol{n}=\mathbf{3}$, node-connected reliability is calculated by dropping fault points .

For $Q_{3}, B_{1}(G)=0, B_{2}(G)=16, B_{3}(G)=34$.

It is not easy to calculated $\boldsymbol{B}_{4}(G)$, so we study $\boldsymbol{B}_{4}(G)$ by the adjacent matrix of $\boldsymbol{Q}_{3}$, the element 1 in the adjacent matrix of $\boldsymbol{Q}_{3}$ indicates that two vertices are connected in $\boldsymbol{Q}_{3}$, and the element 0 in the adjacent matrix of $\boldsymbol{Q}_{3}$ indicates that two vertices are disconnected in $\boldsymbol{Q}_{3}$.

Dropping four vertices of $\boldsymbol{Q}_{3}$ acts as dropping 4 rows and 4 columns simultaneously in the adjacent matrix, which gives rise to a remaining matrix of order 4.There are $\boldsymbol{c}_{\mathbf{8}}^{\mathbf{4}}=\mathbf{7 0}$ different remaining matrices, Among these, the matrix in which the amount of element 1 is less than 3, is disconnected, therefore $\boldsymbol{B}_{\mathbf{4}}(\boldsymbol{G}) \mathbf{3 2}$.

Considering that 3 fault vertices must be adjacent to the same vertices to make the remaining vertices disconnected, it is gained that $\boldsymbol{B}_{5}(G)=\mathbf{8}$.

Because 3- dimensional hypercube is 3-connected, it is definite that the remained vertices become connected graph when the fault points is less than 3.Therefore

$$
B_{6}(G)=0, \quad B_{7}(G)=0, \quad B_{8}(G)=0
$$

It turns out the node-connected reliability

$$
\left.R\left(Q_{3}, p\right)\right)=1-\left\{16 p^{2}(1-p)^{6}+34 p^{3}(1-p)^{5}+32 p^{4}(1-p)^{4}+8 p^{5}(1-p)^{3}\right\}
$$

The node-connected reliability of $\boldsymbol{C Q}_{3}$ is calculated in the same way,

$$
\left.R\left(C Q_{3}, p\right)\right)=1-\left[16 p^{2}(1-p)^{6}+34 p^{3}(1-p)^{5}+26 p^{4}(1-p)^{4}+8 p^{5}(1-p)^{3}\right]
$$

So we obtained $R\left(Q_{3}, p\right)>\boldsymbol{R}\left(C Q_{3}, p\right)$, the reliability of graph $C Q_{3}$ is superior to $Q_{3}$.

If $n \geq \mathbf{4}$, the vertices increase by $\mathbf{2}^{n}$ times .It is difficult to calculate the reliability of graph $\boldsymbol{Q}_{n}$ and $\boldsymbol{C Q}_{n}$, but they are recursive networks from lower dimensional to higher dimensional, we can obtain the lower bound of node-reliability

$$
\begin{aligned}
& R\left(H_{n}, p\right) \geq 1-(1-p)^{\left(2^{n}-2^{3}\right)}\left[16 p^{2}(1-p)^{6}+34 p^{3}(1-p)^{5}+32 p^{4}(1-p)^{4}+8 p^{5}(1-p)\right] \\
& R\left(C Q_{n}, p\right) \geq 1-(1-p)^{\left(2^{n}-2^{3}\right)}\left[16 p^{2}(1-p)^{6}+34 p^{3}(1-p)^{5}+26 p^{4}(1-p)^{4}+8 p^{5}(1-p)\right]
\end{aligned}
$$

It is clear that the lower bound of $\boldsymbol{R}\left(Q_{n}\right)$ is greater than he lower bound of $R\left(C Q_{4}\right)$. 


\section{Conclusions}

We supply the topological property of the two network structures by proving lower bounds of all-terminal reliability and node-connected reliability, and draw a conclusion that the crossed cube is superior to the hypercube in the lower bound of two kinds of reliability.

\section{Acknowledgments}

Email: 13234081901@163.com

\section{References}

[1] Efe K. A variation of the hypercube with lower diameter[J]. IEEE Trans on Computers, 1991,40(1):1312-1316

[2]Leighton F T. Introduction to parallel algorithms and architectures: arrays, trees, hypercubes[M]. California: Morgan Kaufmann Publishers,1992.

[3] Saad Y, Schultz M. Topological properties of hypercube[J]. IEEE Trans on Computers, 1988, 37(7):867-871

[4] COLBOURN C J. The Combinations of Network Reliability[M]. Britain: Oxford University Press, 1987. 